\title{
Case Report: Endoscopic Removal of an Eroded Gastric Band Causing Small Bowel Obstruction upon Migration into the Proximal Jejunum
}

\author{
Amir Sleiman ${ }^{1} \cdot$ Anne Sophie Studer ${ }^{1} \cdot$ Pierre Y. Garneau ${ }^{1} \cdot$ Ronald Denis $^{1} \cdot$ Mark Magdy $^{1} \cdot$ Majed Alanazi $^{1}$. \\ Radu Pescarus ${ }^{1}$
}

Received: 9 June 2020 / Revised: 30 July 2020 / Accepted: 31 July 2020 / Published online: 11 August 2020

(C) Springer Science+Business Media, LLC, part of Springer Nature 2020

\begin{abstract}
Background Adjustable gastric banding (AGB) is on the decline due to its relatively modest amount of expected weight loss, coupled with high rates of revision and complications such as band erosion. Management of eroded gastric bands can be challenging especially when complete intra-gastric erosion is followed by distal migration causing small bowel obstruction.

Methods We present an endoscopic option of using a pediatric colonoscope to remove an eroded AGB causing jejunal obstruction.

Result Endoscopic removal of an eroded ABG causing bowel obstruction was successful.

Conclusion Endoscopy remains a safe and relatively non-invasive approach to deal with such complications.
\end{abstract}

Keywords Adjustable gastric band (AGB) $\cdot$ Band complications $\cdot$ Eroded band $\cdot$ Migrated band $\cdot$ Small bowel obstruction (SBO) . Pediatric colonoscope $\cdot$ Endoscopic removal

\section{Introduction}

Adjustable gastric banding (AGB) is a purely restrictive procedure that involves placing a tight, soft silicone ring around the top part of the stomach. The band is connected to an

Radu Pescarus

radupescarus@gmail.com

Amir Sleiman

amir.sleiman@gmail.com

Anne Sophie Studer

annesophiestuder@yahoo.fr

Pierre Y. Garneau

pierre.y.garneau@sympatico.ca

Ronald Denis

ronalddenis@videotron.ca

Mark Magdy

mark_magdy@hotmail.com

Majed Alanazi

almodyani@hotmail.com

1 Department of Minimally Invasive and Bariatric Surgery, Hôpital du Sacré-Coeur, CIUSSS du Nord de l'île de Montréal, 5400 Boulevard Gouin Ouest H4J 1C5, Québec Montréal, Canada injection port placed in the subcutaneous tissue. Partially responsible for its overall decline, late complications of AGB include band erosion, band slippage or prolapse, port or tubing malfunction, leakage at the port site tubing or band, pouch or esophageal dilatation, and esophagitis [1,2]. It has been estimated that band removal will eventually be necessary in 50$80 \%$ of AGB patients [3]. Long-term results from a series of 78 AGB patients showed that nearly $40 \%$ of patients experienced major complications, $22 \%$ had minor complications, and almost half of the patients required reoperation [4]. Major complications included band erosion (28\%), pouch dilation (11\%), and band infection (1\%). Minor complications included port-tubing disconnections (20\%), incisional hernias $(5 \%)$, and port infections (2\%).

In a series of 571 AGB patients from February 1998 to July 2006, intragastric band migration was found to be an unusual but a major long-term complication of gastric banding: its incidence ranges from 0.5 to $3.8 \%$, and it universally mandates removal of the band [5]. The classic treatment for band erosion has been the laparoscopic removal of the eroded band. Laparoscopic approaches can be challenging due to the potential fibrosis and inflammation surrounding the eroded band as well as the need to perform a gastrotomy. However, the successful endoscopic management of this 
complication has aided in decreasing the need for surgical intervention [6].

In this case report, we describe the use of a pediatric colonoscope to remove an eroded and subsequently migrated AGB causing small bowel obstruction (SBO) in the proximal jejunum. Informed consent was obtained from the patient for publication of this case report and accompanying images.

\section{Clinical Case}

A 69-year-old female presented with worsening abdominal pain over the last 2 days. The colicky and intermittent pain was mostly localized in the epigastric area and was radiating to the back. She denied any nausea, vomiting, fever, or any change in her bowel habitus. Surgical history included an uneventful laparoscopic AGB performed over 10 years ago, and her current BMI is 31.6. Her laboratory work-up was all within normal. A CT of the abdomen and pelvis (Fig. 1a, b) showed an endoluminal band located in the proximal jejunum, disconnected from the intragastric tubing, with no evidence of pneumoperitoneum. The patient was scheduled for surgical intervention; however, due to the COVID-19 pandemic crisis, a 48-h delay in management occurred. During this period, a clinical picture of small bowel obstruction with abdominal distension and vomiting evolved despite persistently normal inflammatory markers.

\section{The Procedure}

The patient underwent endoscopic exploration with a pediatric colonoscope under general anesthesia. The abdomen was prepped in case a conversion to laparoscopy was necessary given the distal location of the AGB. After the aspiration of
$2 \mathrm{~L}$ of fluids from the stomach and duodenum, the jejunum was intubated successfully. A proximal food bezoar and the migrated AGB were identified at about $15 \mathrm{~cm}$ distal to the Treitz (Fig. 2a). The band was then snared by encircling the severed end of the buckle, the point of disconnection between the band and tubing. Under direct endoscopic visualization, the band was retracted into the stomach where it was subsequently sectioned using a gastric band cutter (A.M.I. Gastric Band Cutter System, Agency for Medical Innovation GmbH, Götzis, Austria) (Fig. 2b, c). The sectioned band was then snared and successfully retrieved trans-orally. No luminal injury was noted in the bowel nor in the stomach. Retroflexion of the endoscope did not reveal any fistula within the cardia or fundus of the stomach; however, a small part of the AGB tube was found, and it was also trans-orally retrieved.

Removal of the subcutaneous port was performed through a small $15-\mathrm{mm}$ incision. The complete AGB apparatus is shown here (Fig. 2d). The procedure was completed with a total duration of $75 \mathrm{~min}$ and was well-tolerated by the patient, who was discharged home on the same day on proton pump inhibitor prophylaxis. The patient presented 1 week post surgery with an unrelated colitis for which an outpatient antibiotic treatment was given. Based on 1-month follow-up, the patient is asymptomatic and has recovered well.

\section{Discussion}

Endoscopic management of band erosions together with extraction of the subcutaneous port through a separate small skin incision represents an elegant minimally invasive option. Multiple techniques for intragastric band sectioning and retrieval have been described [7, 8]. Rarely, complete gastric band erosion can be associated with band disconnection and migration into the small bowel. As the band migrates into the

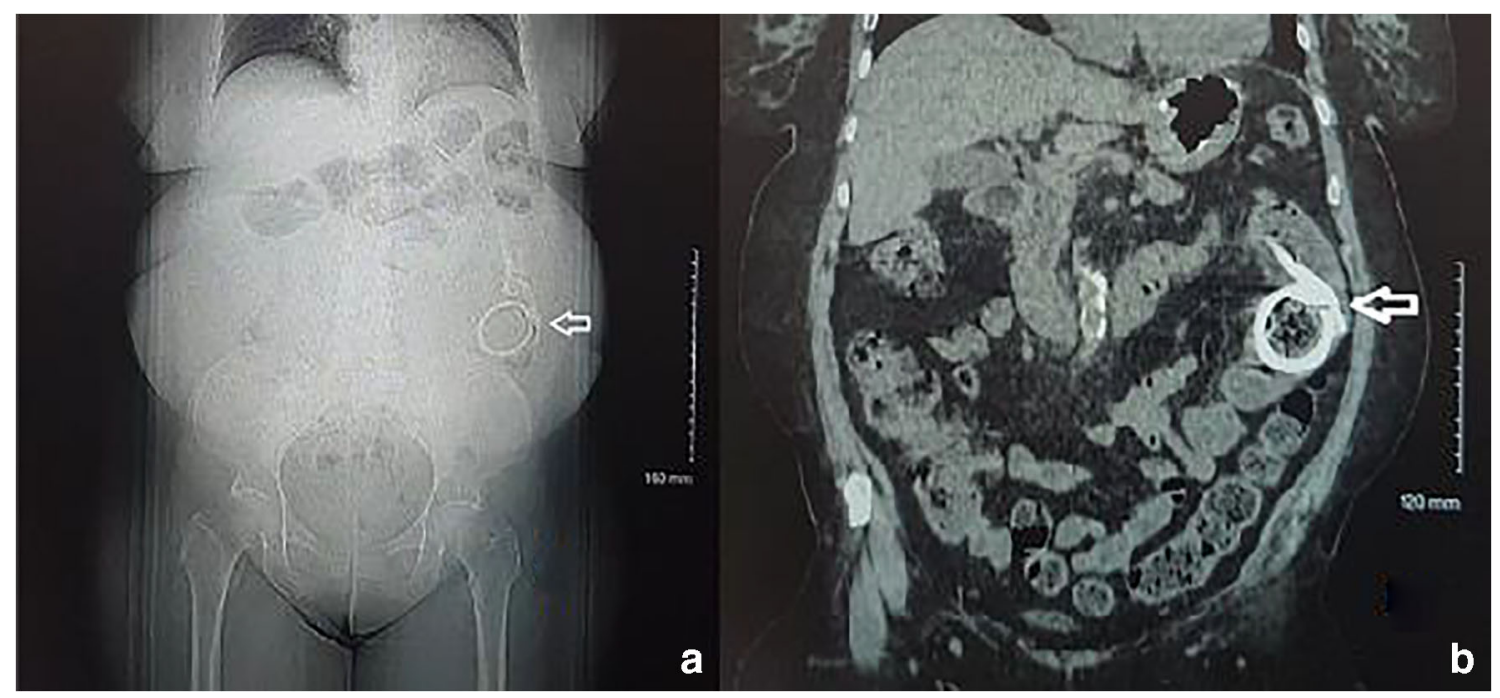

Fig. 1 Arrow showing the eroded and migrated AGB in the jejunum. a Scout of CT and b CT abdomen and pelvis 


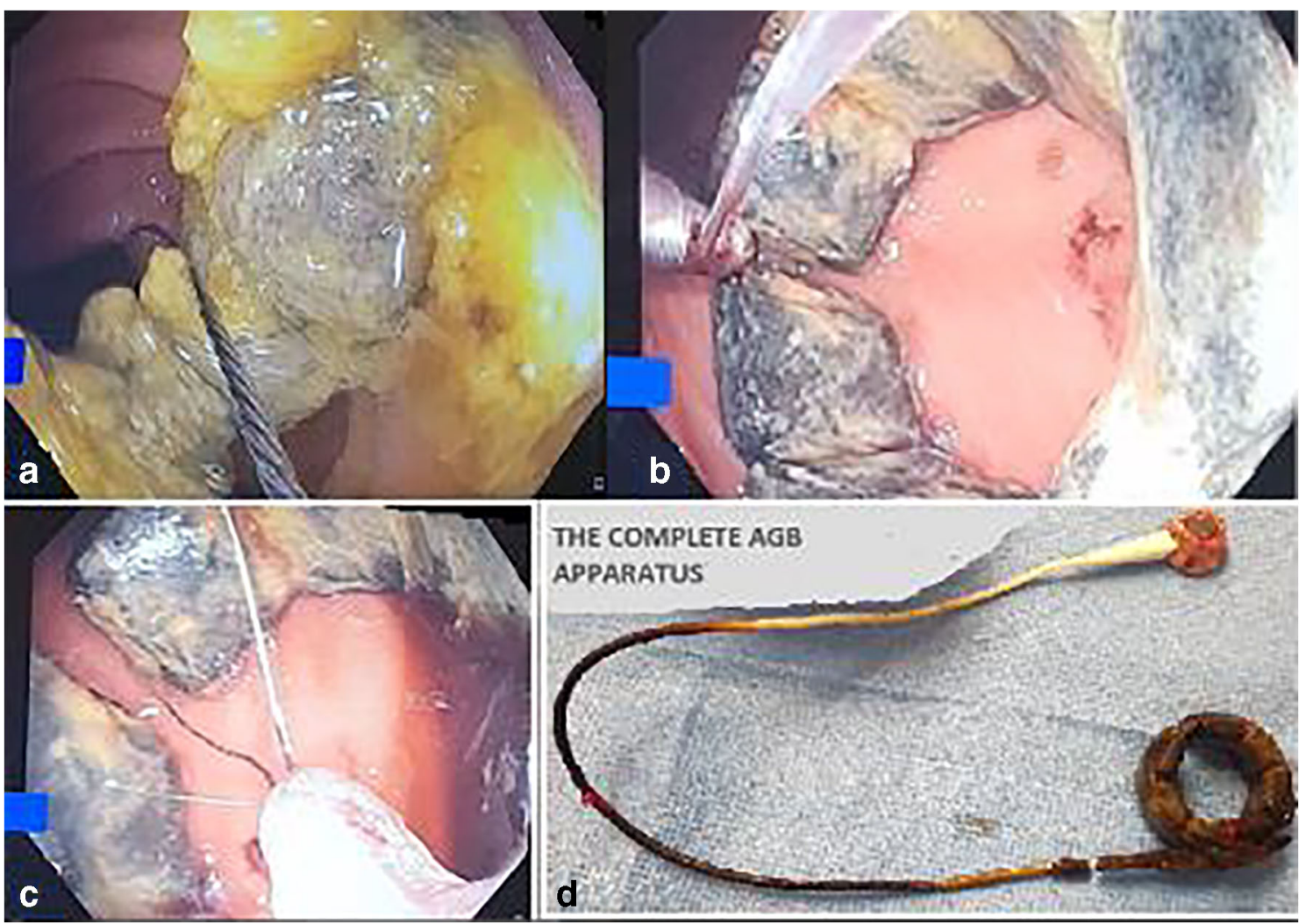

Fig. 2 a The food bezoar, the eroded and migrated AGB being snared from $15 \mathrm{~cm}$ distal to the Treitz and then being placed in the stomach. $\mathbf{b}$ Sectioning of the AGB in the stomach using AMI band cutter. $\mathbf{c}$ Snaring and removal of the AGB from the stomach. $\mathbf{d}$ The complete adjustable gastric band apparatus jejunum, it becomes more challenging to retrieve endoscopically, especially when associated with small bowel obstruction. Using a pediatric colonoscope was befitting and enabled the extension of the endoscopic reach in an adult and the retrieval of the distally migrated band. This technique saved the patient from a surgical procedure in which opening and then suturing of the digestive organs would have been performed, with all its inherent risks and consequences.

\section{Conclusion}

Despite the decline in the laparoscopic AGB procedures, longterm complications of the procedure still arise including complete erosion and migration of the band distally, leading to obstruction. Bariatric surgeons must be aware of these complications and be able to manage them. Endoscopy remains a safe and relatively noninvasive approach to deal with such complications. To our knowledge, this is the first report illustrating the endoscopic removal of an eroded, migrated, and disconnected AGB causing SBO in the proximal jejunum using a pediatric colonoscope. Although potentially challenging, this minimally invasive approach enabled the same day discharge and prevented further unwarranted hospital exposure during the COVID-19 pandemic.

\section{Compliance with Ethical Standards}

Conflict of Interest The authors declare that they have no conflict of interest.

\section{References}

1. DeMaria EJ, Sugerman HJ, Meador JG, et al. High failure rate after laparoscopic adjustable silicone gastric banding for treatment of morbid obesity. Ann Surg. 2001;233:809-18.

2. DeMaria EJ. Laparoscopic adjustable silicone gastric banding: complications. J Laparoendosc Adv Surg Tech A. 2003;13:271-7.

3. Falk V, Sheppard C, Kanji A, et al. The fate of laparoscopic adjustable gastric band removal. Can J Surg. 2019;62(5)

4. Himpens J, Cadière GB, Bazi M, et al. Long-term outcomes of laparoscopic adjustable gastric banding. Arch Surg. 2011;146:802-7.

5. Lattuada E, Zappa MA, Mozzi E, et al. Band erosion following gastric banding: how to treat it. Obes Surg. 2007;17:329-33. 
6. Mozzi E, Lattuada E, Zappa MA, et al. Treatment of band erosion: feasibility and safety of endoscopic band removal. Surg Endosc. 2011;25:39183922.

7. Neto MPG, Ramos AC, Campos JM, et al. Endoscopic removal of an eroded adjustable gastric band: lessons learned after 5 years and 78 cases. Surg Obes Relat Dis. 2010;6(4):423-7.
8. Parmer C, Mamtora S, Balupuri S. Endoscopic removal of intrajujenal migrated gastric band. Surg Obes Relat Dis. 2016;12(9):e75-6.

Publisher's Note Springer Nature remains neutral with regard to jurisdictional claims in published maps and institutional affiliations. 